\title{
Docking studies of Physalis peruviana ethanol extract using molegro virtual docker on insulin tyrosine kinase receptor as antidiabetic agent
}

\author{
Ayik Rosita Puspaningtyas \\ Department of Chemistry, Faculty of Pharmacy, Jember University, Kalimantan Street I No 2 Jember, Indonesia
}

\begin{abstract}
Physalis peruviana Linn in Indonesia was better known as ciplukan, based on information from urban people in Indonesia is as antidiabetic. In the previeous studies, the levels of blood glucose in animals experimental of Physalis peruviana quantified with glucometer and compared with oral antidiabetic drugs gliclazide, showed that Gliclazide was decrease more levels of glucose significantly than ethanol extract of Physalis peruviana. We have done molecular docking using Molegro Virtual Docker (MVD) on ethanol extract of Physalis peruviana and gliclazide to compare between in silico and in vivo studies. Based on studies before the main content of the ethanol extract of Physalis peruviana were withanolide, 4-OH-withanolide, and perulactone. In this study the results showed that gliclazide had been better bond in insulin tyrosine kinase receptor than main content of Physalis peruviana which can be seen from Moldock score 105.217 and Rerank score -68,2931 means that the energy was lower and more stable binding. Moldock Score of main content Physalis peruviana (withanolide, 4-OH-withanolide, and perulactone) were -93.5472; 70.5843; 88.7881, respectively. Rerank score of main content Physalis peruviana (withanolide, 4-OH-withanolide, and perulactone) were -61.5149; $67.5345 ;-65.7979$, respectively. The hydrogen bonds of withanolide, 4-OH-withanolide, perulactone and gliclazide with amino acid of insulin tyrosine kinase receptor were Phe 1186 and Thr 1186. Finally, in the 3D MVD visualization between main content of ethanol extract of Physalis peruviana and gliclazide can be concluded that interaction of gliclazide was more harmonious than main content of ethanol extract Physalis peruviana.
\end{abstract}

Key Words: Physalis peruviana, antidiabetic, insulin tyrosine kinase receptor, gliclazide, molegro virtual docker.

\section{INTRODUCTION}

Indonesia is a tropical country which has much variety of plants and much potential for medicine. On the $20^{\text {th }}$ century, discovery of new drug from plants are increasing. One of the plants is Physalis peruviana Linn which in Indonesia known as "ciplukan". Based on information from urban people that this plant used as antidiabetic agents (Ahmed et al., 2012; Chang et al., 2008; Cirigliano et al., 2008; Marin et al., 2010; Yen et al., 2010).

Several studies showed that oral antidiabetic drugs in the market give effect on kidneys and liver. For example the effect of consuming gliclazide, in some studies is hypoglycemic, while other side effects were related to gastrointestinal, skin, hematologic, central nervous system and eye symptoms (Nogrady and Weaver, 2005). Some research about $P$. peruviana published in Changin International Journal in 2008 showed that extract of $P$. peruviana, herbs provide therapeutic activities as antihepatitis disease, cancer, asthma, inflammatory, dermatitis, reumatis, and antimalarial. The activity of $P$. peruviana herb extract as antidiabetic by in vivo test was studied and will be compared with in silico study (Ramadan, 2001; Runyoro, 2006). Based on studies before the main content of ethanol extract of $P$. peruviana, were withanolide, 4-OH-withanolide, and perulactone (Fang et al., 2012; Kuang et al., 2011; Saeed et al., 1999).

Studies on insulin tyrosine kinase will be increasing, although insulin tyrosine kinase does not represent a new target in drug development of antidiabetic. This study aims the rational target and effective drug design to find

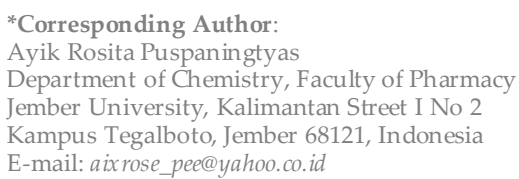

antidiabetic agent. Currently, molecular docking approach has been used in modern drug design to understand drug-receptor interactions. According to the literature, computational techniques can support and help in making drug design. In addition, it can be used to know mechanism of drug-receptor interactions. In this study the researcher docked insulin tyrosine kinase with main content of ethanol extract of $P$. peruviana as ligand by Molegro Virtual Docker (MVD) (Hwang et al., 2012; Molegro Virtual Docker, 2010; Naeem et al., 2013; Nogrady and Weaver, 2005).

Molecular docking is a computer-assisted based on drug design. The purpose of receptor and ligand docking can be predicted the ligand binding model threedimensional visualization. This research was conducted to understand the interaction of three main contents of $P$. peruviana that bind to receptors. Ligand was consisted of ethanol extracts of $P$. peruviana (withanolide, 4-OHwithanolide, and perulactone) as active compounds compared with gliclazide as control in insulin tyrosine kinase receptor (Singh et al., 2012; Singh and Konwar, 2012; Thomsen and Christensen, 2006; Thomsen and Christensen, 2009).

\section{MATERIALS AND METHODS}

\section{Hardware and software}

Molegro Virtual Docker 5.02010 and [Sybil2] were used for molecular modeling with dual core processors.

\section{Molecular structure and optimization}

The structure of main contents of ethanol extract of $P$. peruviana (withanolide, 4-OH-withanolide, and perulactone), it was drawn by ChemBioDrawUltra11.0. Geometrical isomers structure of main content ofethanol extract of $P$. peruviana (withanolide, 4-OH-withanolide, and perulactone) was further optimized by ChemBio- 
Draw3D Ultra v11.0. The structure of insulin tyrosine kinase receptor (PDB code 2B4S) was obtained from the Protein Data Bank (http://www.rcsb.org). Molecular Docking molecules of main contents of ethanol extract of $P$. peruviana to active site of insulin tyrosine kinase, it was done by Molegro Virtual Docker program after saved in Sybil2. (http://www.molegro.com). These algorithms use a cavity detection algorithm (cavity) to detect potential binding sites receptor which have active region to bind of drug (ligand). Screening of the most stable form of ligand combined with MM2 as energy minimize to get conformational search for generating ligand poses consistent in active form of receptor (insulin tyrosine kinase). Candidate poses were minimized incurrent energy use gridbase method for evaluating receptor-ligand interaction energy was saved in Mol2. Docking was done by default on non-ligand Molegro Virtual Docker with 10 cavity but we were used cavity 1 (1367.04 volumes) for insulin tyrosin kinase with a grid resolution of $0: 30$ with maximum iteration parameters is 1500 , maximum population size is 50 and pose of energy generation is 100.00. After all, it used simplex evolutionary step 300 and max distance factor 1.00 (Molegro Virtual Docker, 2010; Thomsen and Christensen, 2006; Thomsen and Christensen, 2009).

\section{Function Assessment (Score)}

Conformational forms which had been further docked and gave different scoring functions available in [Sybil2]. Molegro Virtual Docker algorithm use internal valuation function which DockScore was used to select and redifferentiate position for each compounds. DockScore is simple style on field assessment of functions that estimate energy interaction toward summing energy interaction of ligand/receptor and internal energy of ligand. Magnetic field on Molegro Virtual Docker was used to solve electrostatic parameters in DockScore. Putative position used 3D structure to produce a score that was saved Sybil2 file type. Each docking was minimized by pure DockScore, molecular mechanics of evaluation function and pose which was used and presented in this study such as Rerank Score, RMSD (Root Mean Standard Deviation) and $\mathrm{H}$ Bond. Figure 1 shows the 3D structure was saved in Sybil2 (Molegro Virtual Docker, 2010; Thomsen and Christensen, 2006; Thomsen and Christensen, 2009).

\section{Protein}

Protein structure of insulin tyrosine kinase (PDB code 2B4S) combine with high-resolution classification $(2.3 \AA)$ X-rays with PTP1B complexes and ligands were imported into Sybil2 which is extracted to leave a cavity. Simulations performed with and without ligand binding to explain the role of insulin tyrosine kinase of main content of the ethanol extract of $P$. peruviana (withanolide, 4-OHwithanolide, and perulactone). This docking was not used in presence of water.

\section{RESULTS AND DISCUSSION}

Crystal structure of insulin tyrosine kinase receptor was involving transferase receptor classification in complex with native ligand PTPIB (2B4S) which gives information about the location, composition and functional conformation opportunity of receptor binding space. In this study, we used X-ray structure of the ternary complex insulin tyrosine kinase in native ligand 2B4S (PDB code 2B4S) in docking.

\section{Validation of docking methods}

Validation of docking was used to ensure orientation and position of ligand binding that was obtained from docking studies by MVD program with ten replication of each running. And then the parameters must be validated in crystal structure of receptor (PDB code 2B4S). Native ligand $2 \mathrm{~B} 4 \mathrm{~S}$ in insulin tyrosine kinase was found in complex with ligand-receptor conformation. After that, it was extracted and put it into appropriate active site for ligand binding to determine the ability oforientation and position of ligand that was observed in cavity ligandreceptor crystal structure. The results, indicate that control docking has optimal orientation of ligand complex binding by Molegro Virtual Docker program, it was able to determine toward active 2B4Swhich consist of 10 atoms (Figure 3), in cavity 1 (1367.04 volumes) and use protein B (2B4S) of insulin tyrosin kinase structure crystalline. The methods can be said valid if RMSD of parameters docking showed minimal value.

Interaction model between main content of ethanol extract of $P$. peruviana (withanolide, 4-OH-withanolide, perulactone) and insulin tyrosine kinase receptor were presented at Figure 4. Molecular docking model of main content of ethanol extract of $P$. peruviana (withanolide, 4$\mathrm{OH}$-withanolide, and perulactone) in active site insulin tyrosine kinase of intermolecular flexible docking simulations was obtained through Molegro Virtual Docker program. Dockscore and Rerank Score were calculated from stable conformation of insulin tyrosine kinase complex.

All compounds in data set were incorporated in active site of insulin tyrosine kinase, and used the same protocol. The insulin tyrosine kinase consists of four sites. They were A, B, C and D proteins and which applied here was B protein. Insulin tyrosine kinase is polymer that consist of fold $\alpha$ helix and $\beta$ sheet. The $\alpha$ helix and $\beta$ sheet of stack has monomers form with dimer interface. Dimer contains two active sites, one of them was in each monomer. This can be seen at Figure 4 insulin tyrosine kinase with main content of ethanol extract of $P$. peruviana (withanolide, 4-OH-withanolide, and perulactone).

Binding between active site insulin tyrosine kinase and main content of ethanol extract of $P$. peruviana (withanolide, 4-OH-withanolide, perulactone) on Phe 1186 and Thr 1186 amino acids showed in Figure 5 and Table 1, it showed the formation of hydrogen bonds of insulin tyrosine kinase with Phe 1186 and Thr 1186 was also showed.

The most of amino acid residues in hydrophobic active site were involved in affinity hydrophobic bonding interactions of ligand (main content of ethanol extract of $P$. peruviana (withanolide, 4-OH-withanolide, perulactone) and gliclazide) (Figure 6). This figure shows hydrophobic and electrostatic bonding between main content of ethanol extract of $P$. peruviana (withanolide, 4-OHwithanolide, perulactone) and gliclazide with insulin tyrosine kinase receptor.

The structure of most main content of ethanol extract of $P$. peruviana (withanolide, 4-OH-withanolide, perulactone) was nonpolar, so this gave an advantage to hydrophobic for binding inside chains as receptor active site. The main content of ethanol extract of $P$. peruviana (withanolide, 4-OH-withanolide, perulactone) has similar role binding with gliclazide that has been proven as antidiabetic agent and occupied in cavity 1 of insulin tyrosine kinase receptor toward hydrophobic binding with aromatic ring, aliphatic chain and carbonyl group. Aromatic ring was important for binding, on the other 
Table 1: Hydrogen bonding interactions between withanolide, 4-OH-withanolide, perulactone and gliclazide with insulin tyrosine kinase receptor.

\begin{tabular}{ccc}
\hline Ligand & Thr 1186 & Phe 1186 \\
\hline Withanolide & + & + \\
4-OH- Withanolide & + & + \\
Perulactone & + & + \\
Gliclazide & + & + \\
\hline
\end{tabular}

Table 3: Physicochemical parameters of withanolide, 4-OHwithanolide, perulactone and gliclazide.

\begin{tabular}{ccccc}
\hline Ligand & LogP & ClogP & MR & CMR \\
\hline Withanolide & 7.63 & 9.137 & 122.95 & 12.4656 \\
4-OH- Withanolide & 0.63 & -0.123701 & 130.2 & 13.1906 \\
Perulactone & 7.66 & 9.117 & 122.86 & 12.4656 \\
Gliclazide & 1.97 & 1.093 & 84.53 & 8.5223 \\
\hline
\end{tabular}

Note: $\log P, C \log P, M R$, and CMR value calculated by ChemBioDraw Ultra 11.0
Table 2: Docking parameters in active site of insulin tyrosine kinase receptor.

\begin{tabular}{ccccc}
\hline Ligand & $\begin{array}{c}\text { MolDock } \\
\text { Score }\end{array}$ & $\begin{array}{c}\text { Rerank } \\
\text { Score }\end{array}$ & RMSD & HBond \\
\hline Withanolide & -93.5472 & -61.5149 & 14.9808 & 0.894142 \\
4-OH- Withanolide & -70.5843 & -67.5345 & 10.6962 & -17.9847 \\
Perulactone & -88.7881 & -65.7979 & 13.8455 & -2.60845 \\
Gliclazide & -105.217 & -68.2931 & 11.9351 & -7.05117 \\
\hline
\end{tabular}

Table 4: Comparison between in vivo activities of Physalis peruviana ethanol extract and gliclazide.

\begin{tabular}{cc}
\hline Compounds & IC 50 \\
\hline Phylanti extract & $219.71 \mathrm{mg} / \mathrm{kgBB}$ \\
Gliclazide & $8 \mathrm{mg} / 70 \mathrm{kgBB}$ \\
\hline
\end{tabular}

(a)<smiles>C[C@H]1C[C@H]([C@@H](C)[C@H]2CC[C@H]3[C@@H]4CC[C@H]5CCCC[C@]5(C)[C@H]4CC[C@]32C)OC(=O)[C@H]1C</smiles>

(b)<smiles></smiles><smiles>C[C@@H](CC[C@H]1COC(=O)[C@@H]1C)[C@H]1CC[C@H]2[C@@H]3CC[C@@H]4CCCC[C@]4(C)[C@H]3CC[C@@]21C</smiles>

(c)

Figure 1: Chemical structure, (a) withanolide, (b) 4-OH-withanolide, (c) perulactone, (d) gliclazide.

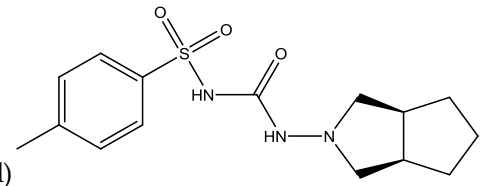

(d)

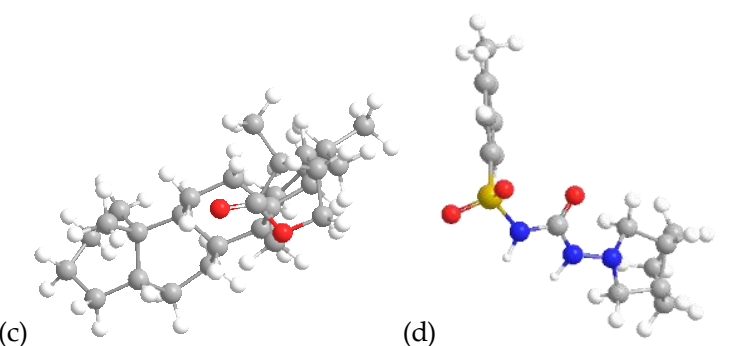

(b)

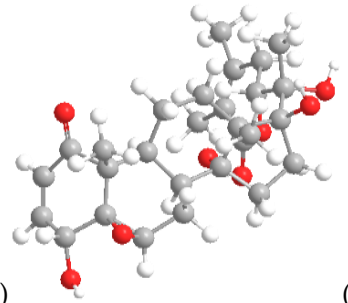

(c)

(d)

Figure 2: 3D structure of (a) withanolide, (b) 4-OH-withanolide, (c) perulactone, (d) gliclazide.

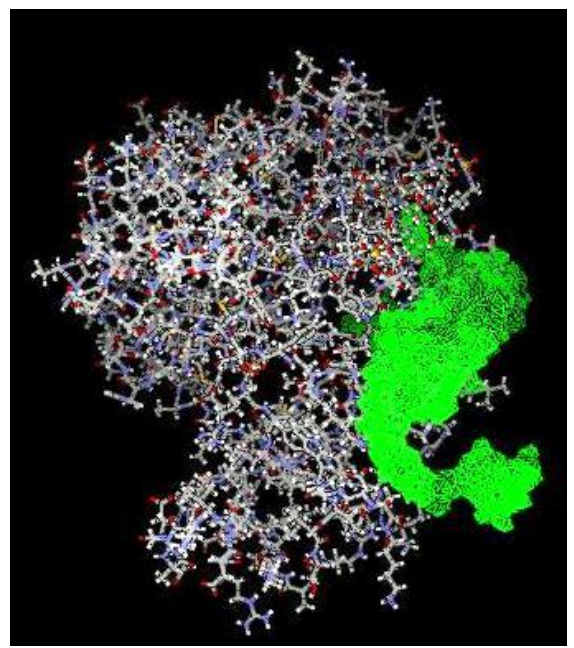

Figure 3: Crystal structure of insulin tyrosine

kinase complex that binds to ligand in the cavity (green). 


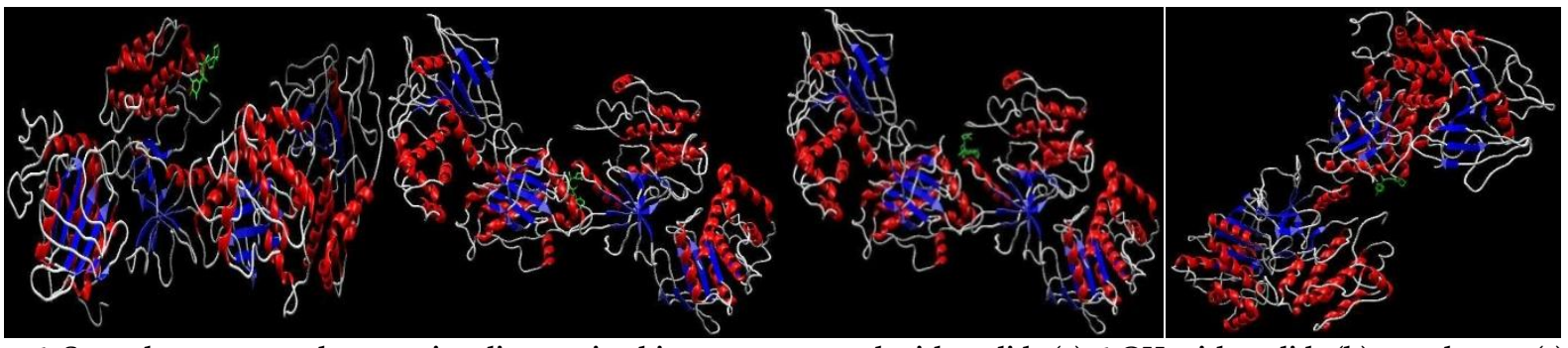

Figure 4: Secondary structure between insulin tyrosine kinase receptor and withanolide (a), 4-OH-withanolide (b), perulactone(c) \& gliclazide (d).

$$
\text { (a) }
$$
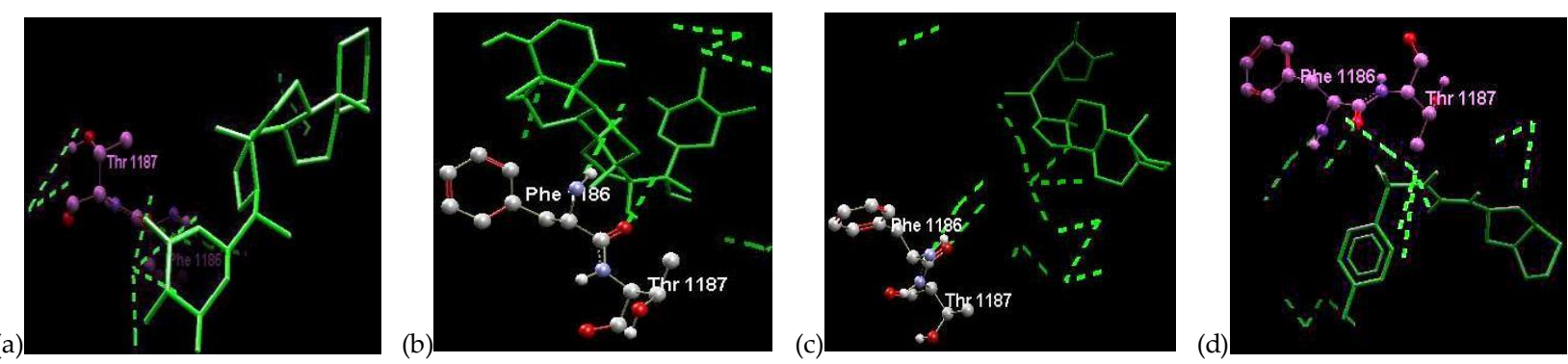

Figure 5: Hydrogen bonding interactions between insulin tyrosine kinase receptor and withanolide (a), 4-OH-withanolide (b), perulactone(c) \& gliclazide (d).
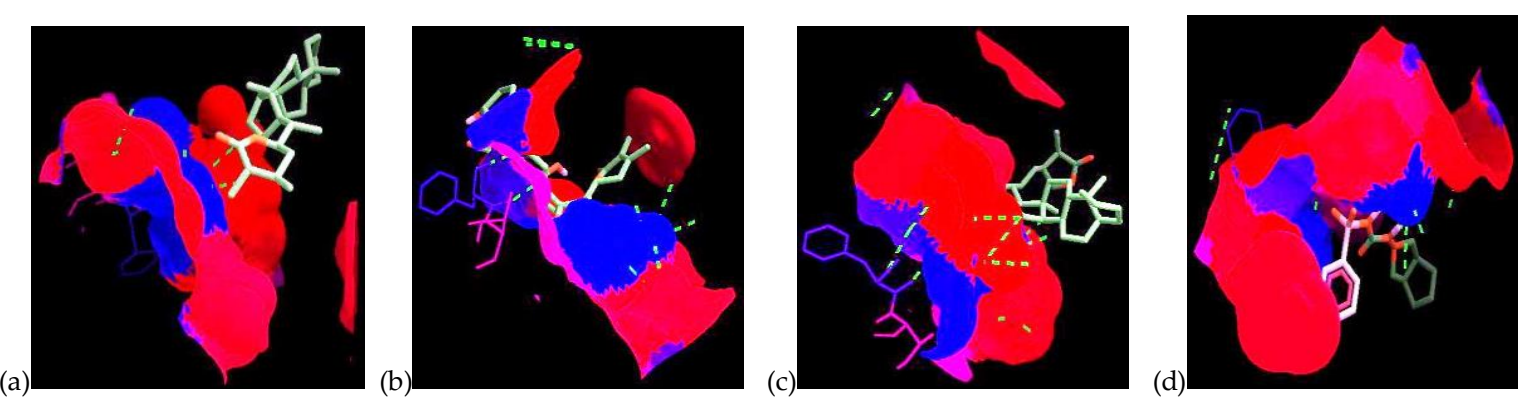

Figure 6: Hydrophobic bonding interactions between insulin tyrosine kinase and withanolide (a), 4-OH-withanolide (b), perulactone(c) \& gliclazide (d).
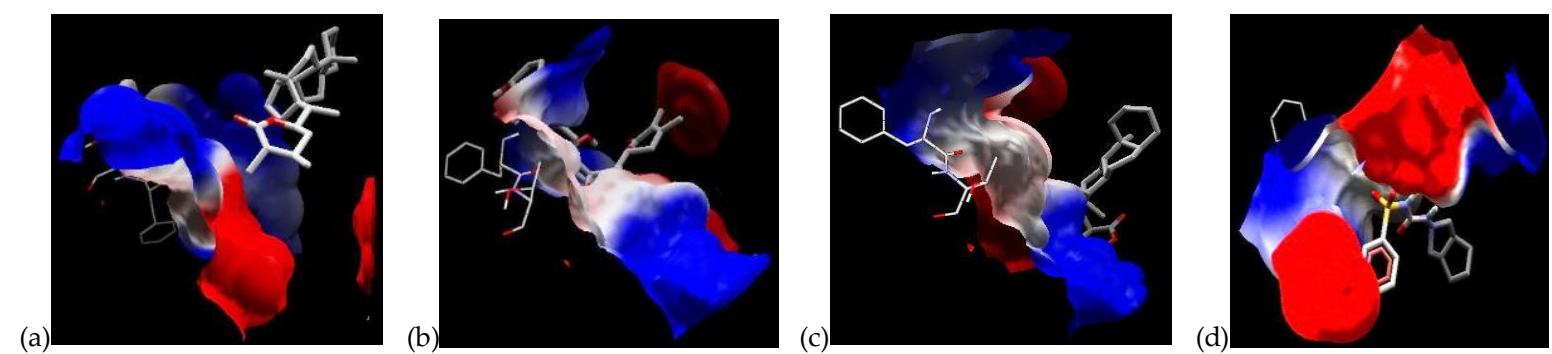

Figure 7. Electrostatic bonding interactions between insulin tyrosine kinase and withanolide (a), 4-OH-withanolide (b), perulactone(c)) \& gliclazide (d).
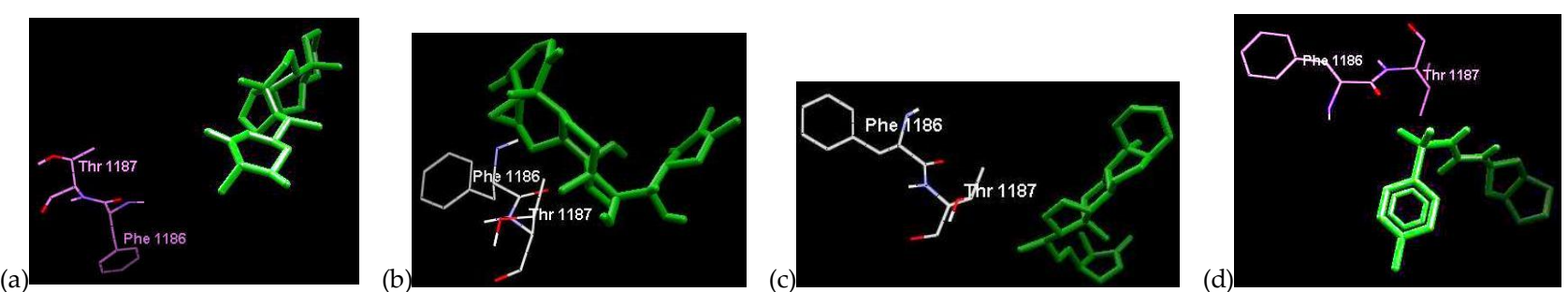

Figure 8: Pose organize between insulin tyrosine kinase and withanolide (a), 4-OH-withanolide (b), perulactone(c) \& gliclazide (d) 
The structure of most main content of ethanol extract of $P$. peruviana (withanolide, 4-OH-withanolide, perulactone) was nonpolar, so this gave an advantage to hydrophobic for binding inside chains as receptor active site. The main content of ethanol extract of $P$. peruviana (withanolide, 4$\mathrm{OH}$-withanolide, perulactone) has similar role binding with gliclazide that has been proven as antidiabetic agent and occupied in cavity 1 of insulin tyrosine kinase receptor toward hydrophobic binding with aromatic ring, aliphatic chain and carbonyl group. Aromatic ring was important for binding, on the other side the main content of ethanol extract of $P$. peruviana (withanolide, 4-OHwithanolide, perulactone) can not binding well in active site of insulin tyrosine kinase because it didn't have aromatic ring. The docking studies of main content of ethanol extract of $P$. peruviana (withanolide, 4-OHwithanolide, perulactone) in active site of insulin tyrosine kinase receptor showed that MolDock Score and Rerank Score were lower than gliclazide. Decreasing interaction has an effect on the result of decreasing affinity binding between ligand-receptor. The correlation between docking scores and activities were shown by Dock Score, Rerank scores and physicochemical parameters of main content of ethanol extract of $P$. peruviana (withanolide, 4-OHwithanolide, perulactone) and gliclazide (Tables 2 and 3).

The value of MolDock Score, Rerank Score, and physicochemical parameters showed that main content of ethanol extract of $P$. peruviana (withanolide, 4-OHwithanolide, perulactone) was lower energy than gliclazide especially 4-OH withanolide. All compounds showed that gliclazide was higher stability and alignment than main content of ethanol extract of P. peruviana (Fang et al., 2012; Kuang et al., 2011; Molegro Virtual Docker, 2010; Naeem et al., 2013; Ramadan, 2001; Runyoro, 2006; Saeed et al., 1999; Yen et al., 2010).

According to physicochemistry parameters especially $\log \mathrm{P}$ (hydrophobic parameters) of main content of ethanol extract of $P$. peruviana (withanolide, 4-OHwithanolide, perulactone) was higher than gliclazide but steric parameters also higher value, it can effect total score of activity (Table 3). Molegro Virtual Docker program can dock for drug design to predict activity in insulin tyrosine kinase receptor as antidiabetic agent toward conformation of binding of ligand and receptor in scores value. Scores of them indicated that gliclazide was substituted aromatic and carbonyl group which was better binding than main content of ethanol extract of $P$. peruviana (withanolide, 4$\mathrm{OH}$-withanolide, perulactone), it can be seen from Moldock and Rerank score (Molegro Virtual Docker, 2010; Thomsen and Christensen, 2006; Thomsen and Christensen, 2009). These results were consistent with $3 \mathrm{D}$ visualization where main content of ethanol extract of $P$. peruviana (withanolide, 4-OH-withanolide, perulactone) was lower interaction of hydrogen, hydrophobic and electrostatic binding than gliclazide in receptor. These result were compared with in vivo study where gliclazide was higher activity than main content of ethanol extract of $P$. peruviana (withanolide, 4-OH-withanolide, perulactone) (Table 4).

The results showed that interaction of gliclazide was more harmonious than ethanol extract of $P$. peruviana (withanolide, 4-OH-withanolide, perulactone) in insulin tyrosine kinase receptor as antidiabetic agent which was supported with 3D visualization, MolDock Score, Rerank Score, and physicochemistry parameters by MVD program.

\section{CONCLUSION}

In this study the results was obtained that gliclazide has better bond in insulin tyrosine kinase receptor as antidiabetic agent than main content of ethanol extract of $P$. peruviana (withanolide, 4-OH-withanolide, perulactone) which can be seen Moldock score 105.217 and Rerankscore68.2931. It means, if it had lower energy so it was more stable in binding. The in vivo test showed that ethanol extract of $P$. peruviana activity was lower than gliclazide. It also supported by hydrogen bonds interaction with Phe 1186 and Thr 1186 amino acids. Finally, interaction of gliclazideis more harmonious than ethanol extract of $P$. peruviana in insulin tyrosine kinase receptor.

\section{ACKNOWLEDGEMENT}

Author is thankful to Prof. Siswandono, MS. Apt and Hadi Poerwono, MSc, Apt, Ph.D for their encouragement, permission and support in computational/software facilities at the Department of Pharmaceutical Chemistry, Airlangga University.

\section{REFERENCES}

Ahmed E. Moneim A and El-Deib K.M. (2012).The Possible protective effects of Physalis peruviana on Carbon Tetrachloride-induced Nephrotoxicity in Male Albino Rats, Life Science Journal; 9(3)

Chang J.C, Lin C.C, Wu S.J, Lin D.L, Wang S.S, Miaw C.Land Ng L.T. (2008) Antioxidative and Hepatoprotective Effects of Physalis peruviana Extract against Ace ta minophen-Induced Liver Injury in Rats, Pharmaceutical Biology, Vol. 46, Nos. 10-11, pp. 724-731

Cirigliano A, Colomirano I, Mareggiani G, and Bado S,. (2008). Biological Effects Of Physalis peruviana L (Solanaceae) Crude Extracts And Its Major Withanolides On Ceratitis Capita ta Wiedemenn (Diptera: Tephritidae), Bolsan veg Plagas 34: 509-515

Fang S, Liu J, and Li B,. (2012). Ten new withanolides from Physalis peruviana, Steroids 77: 36-44. [DOI]

Hwang S.W, Lee J, Shin J, Lee J.Y, Lee K, and Jang D.S. (2012). Inhibitory Effects of Phenylpropanoids Isolated from the Bark of Ailanthus altissima on COX-2 Activity, Bull. Korean Chem. Soc, Vol. 33, No. 8 2759. [DOI]

Kuang H, Yang B, Xia Y and Wang Q,. (2011). Two New Withanolide Lactones from Flos Daturae, Molecules, 16: 5833-5839. [DOI]

Marin A,Z.T, Cortes R, and M, Montoya C, O.I,. (2010). Capegooseberry (Physalis peruviana L) Colombian Ecotype Minimally Processed Inoculated With Native Strain Lactobacillus Plantarum LPMB10 By Means of Vaccum Impregnation Technique, Rev ChilNutrVol 37 no 4

Molegro Virtual Docker, www.molegro.com, Accessed September 25th 2010

Naeem S, Hylands P, and Barlow D,. (2013). Docking Studies of Chlorogenic Acid against Aldose Redutcase by using Molegro Virtual Docker Software, Journal of Applied Pharmaceutical Science Vol. 3(01), pp.013-020

Nogrady T., and Weaver, D.F., (2005). Medicinal Chemistry: A Molecular and Biochemical Approach, 3th ed., Oxford Unive rsityPress, Inc., Oxford, p.485. [DOI]

Ramadan M.F,. (2011). Bioactive phytochemicals, nutritional value, and functional properties of cape gooseberry (Physalis peruviana): An overview, Food Research Internationa 144: 1830-1836. [DOI]

Runyoro, D., (2006), Screening of Tanzanian Medicinal Plants for Anti Candida Activity, Bio Med Central Complementary and alternative Medicine Volume 6

Saeed A, Yasmin R, and Malik A,. (1999). New Withanolide Glycosides from Physalis peruviana L., Chem. Pharm. Bull. 47(4) 477-480

Singh S.P, Bezbaruah R.L, and Bora T.C. (2012). In silico studies of 2-methylheptyl isonicotinate produced by Streptomyces sps. 201 against dihydrodipicolinate synthase enzyme of Mycobacterium tuberculosis, Journal of Biophysical Chemistry Vol.3, No.3, 233-23

Singh S.P and Konwar B.K. (2012). Molecular docking studies of que rcetin and its ana logues aga inst human inducible nitric oxide synthase, SpringerPlus, 1:69. [DOI]

Thomsen R, and Christensen M.H,. (2006). MolDock: A new technique for highaccuracy docking. J Med Chem, 49:3315-3321. [DOI]

Thomsen, R.; and Christensen, M.H. (2009). Molegro Virtual Docker 4.0 User Manual; Molegro ApS: Aarhus, Denmark p.125-133

Yen C, Chiu C, Chang F, Chen J.Y, Hwang C, Hseu C, Yang H, Lee A.Y, Tsai M, Guo Z, Cheng Y, Liu Y, Lan Y, Chang Y, Ko Y, Chang H, and Wu Y. (2010). $4 \beta$ Hydroxywithanolide E from Physalis peruviana (golden berry) inhibits growth of human lung cancer cells through DNA damage, apoptosis and G2/M arrest, BMC Cancer 10:46. 